\title{
Neutron background studies for direct dark matter searches in LSM
}

\author{
V.Yu. Kozlov* for the Edelweiss collaboration \\ Karlsruhe Institute of Technology, Institut für Kernphysik, Postfach 3640, 76021 Karlsruhe, \\ Germany \\ E-mail: Valentin.Kozlovakit.edu
}

One of the most promising particle candidates for dark matter is the supersymmetric neutralino or more generally a weakly interacting massive particle (WIMP). The expected interaction rate of WIMPs in direct search experiments is below 0.01 events/(kg day), thus rising up the importance of having a detailed understanding of potential background. Ambient and muon-induced neutrons constitute a prominent background component. Detailed studies carried out by the EDELWEISS collaboration in this respect are presented. This activity includes dedicated calibrations with neutron sources, monitoring the neutron flux with ${ }^{3} \mathrm{He}$ detectors and measurements with a neutron counter based on Gd-loaded liquid scintillator as well as corresponding MC simulations with full event topology. The impact of the neutron background on current EDELWEISS-2 data-taking as well as for next generation experiments such as EURECA is briefly discussed.

Identification of Dark Matter 2010-IDM2010

July 26-30, 2010

Montpellier France

*Speaker. 


\section{Introduction}

A dark matter search principle of vast direct detection experiments is based on measuring the energy of a nuclear recoil resulting from the WIMP - nucleus elastic scattering. The main challenge then is to understand and reduce all background components in the corresponding energy domain. Since $\gamma$ and $\beta$ particles lead to electron recoils while scattered WIMPs to nuclear recoils, a frequently used strategy is to distinguish between the two types of recoils. This approach, however, does not work when one deals with neutrons of energies relevant to mimic a WIMP event, e.g. of the order of several 100's keV in the case of a Germanium target. Such neutrons appear in an underground laboratory either from natural radioactivity, e.g. U/Th contamination and $(\alpha, \mathrm{n})$ reactions in a rock, concrete and shielding materials, or being produced either directly by remaining muons or in muoninduced electromagnetic and hadronic showers.

\section{EDELWEISS-2 direct dark matter search experiment}

The EDELWEISS-2 experiment, described elsewhere [1], is installed in the $\mathrm{LSM}^{1}$ underground laboratory and uses Ge-bolometers with interleaved electrodes [2] to search for galactic WIMPs. These detectors, once cooled down to $18 \mathrm{mK}$, allow to measure simultaneously heat and ionization signals, the latter one being quenched in case of nuclear recoils. Thus by comparing the two signals it is possible to reach a very high rejection power against $\gamma / \beta$ background [2]. The central part of the set-up (Fig. 1) is a dilution refrigerator which is able to host up to $40 \mathrm{~kg}$ of the detectors. It is surrounded by a lead shield of $20 \mathrm{~cm}$ to protect the bolometers from an external $\gamma / \beta$ radioactivity. The $\mathrm{Pb}$ layer is followed by $50 \mathrm{~cm}$ of polyethylene to moderate neutrons. The muon veto to tag remaining muons completes the installation. Several other detectors for background studies are operated close to the main set-up. Among them are ${ }^{3} \mathrm{He}$ proportional counters to measure ambient neutrons and the neutron counter based on $1 \mathrm{~m}^{3}$ of Gd-loaded liquid scintillator to investigate muon-induced neutrons (Fig. 1). As the current data analysis for dark matter search shows, about a quarter of the total background in EDELWEISS comes from neutrons, thus raising up the importance of the detail knowledge of this background. This becomes even more prominent for a next generation dark matter experiments, e.g. EURECA [3], aiming for a factor of 100 better sensitivity than EDELWEISS-2.

\section{Measurement of ambient neutrons}

${ }^{3} \mathrm{He}$ proportional counters are filled with $400 \mathrm{kPa}$ of ${ }^{3} \mathrm{He}$ and $500 \mathrm{kPa}$ of ${ }^{40} \mathrm{Ar}$ as working gas and make use of a large cross-section for thermal neutron capture on ${ }^{3} \mathrm{He}(\sigma=5333 \pm 7$ barn $)$. By means of these detectors both fast and thermal components of ambient neutrons can be measured: in the former case the counters are surrounded by a massive block of polyethylene $\left(12 \times 20 \times 100 \mathrm{~cm}^{3}\right)$ while in the latter one the naked detectors are used. The measurement campaign of fast neutrons showed that their flux close to the EDELWEISS-2 experiment can be influenced by opening of the massive polyethylene shield of the main set-up (Fig. 1). When the periods with fixed geometry are selected, an average flux of $5 \cdot 10^{-6} \mathrm{~cm}^{-2} \mathrm{~s}^{-1}$ is obtained, being consistent with earlier measurements [4]. For a proper interpretation of results of a dark matter experiment not only the absolute background

\footnotetext{
${ }^{1}$ Laboratoire Souterrain de Modane, France
} 


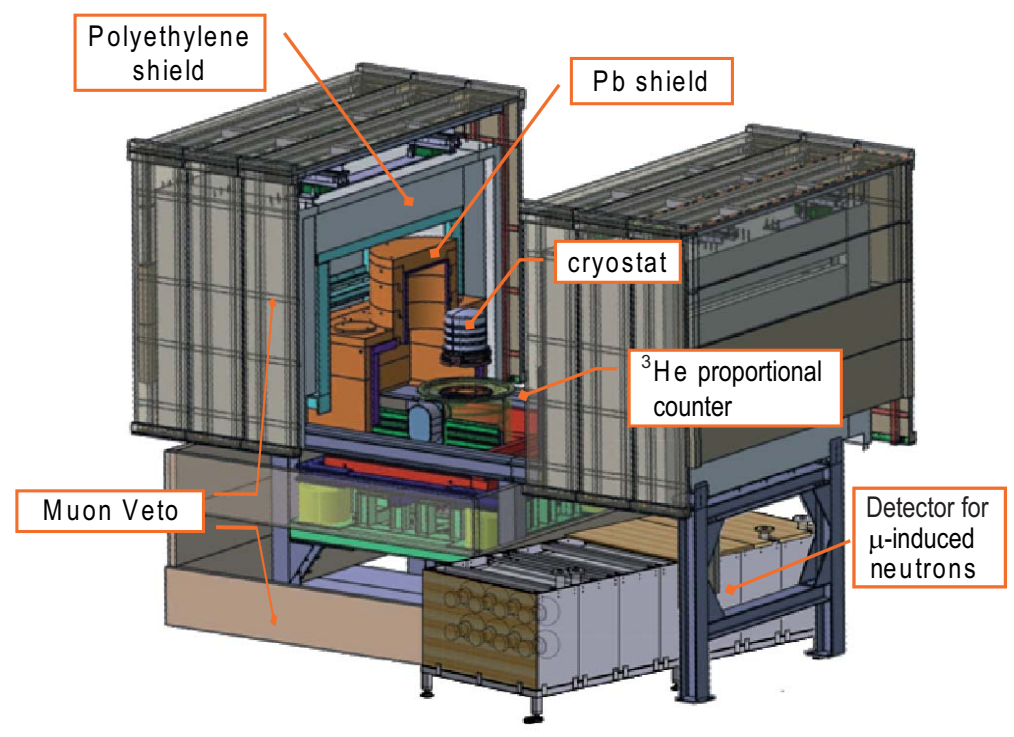

Figure 1: General layout of the EDELWEISS-2 experimental set-up with additional ${ }^{3} \mathrm{He}$ proportional counter for thermal neutrons and liquid scintillator detector to measure muon-induced neutrons.

rate but also its temporal variation is of importance. Preliminary results comparing the fast neutron flux measured through 2008 set an upper limit on an annual variation of less than 4\% (90\% C.L.). Similar measurements performed for thermal neutrons through winter 2008 to summer 2009 show neither seasonal fluctuations nor day-night effect [5]. The absolute flux of thermal neutrons was measured also at different places in the LSM underground laboratory and a variation of up to a factor of 3 was found [5]. This variation can be explained by influence of large neutron shields present in the laboratory (e.g. of EDELWEISS and NEMO-3 experiments) and a different water content and U/Th contamination in the materials close to the ${ }^{3} \mathrm{He}$ counter. The thermal neutron flux measured near the EDELWEISS-2 set-up was found to be $\Phi=\left(3.57 \pm 0.05^{\text {stat }} \pm 0.27^{\text {syst }}\right) 10^{-6} \mathrm{~cm}^{-2} \mathrm{~s}^{-1}$ [5].
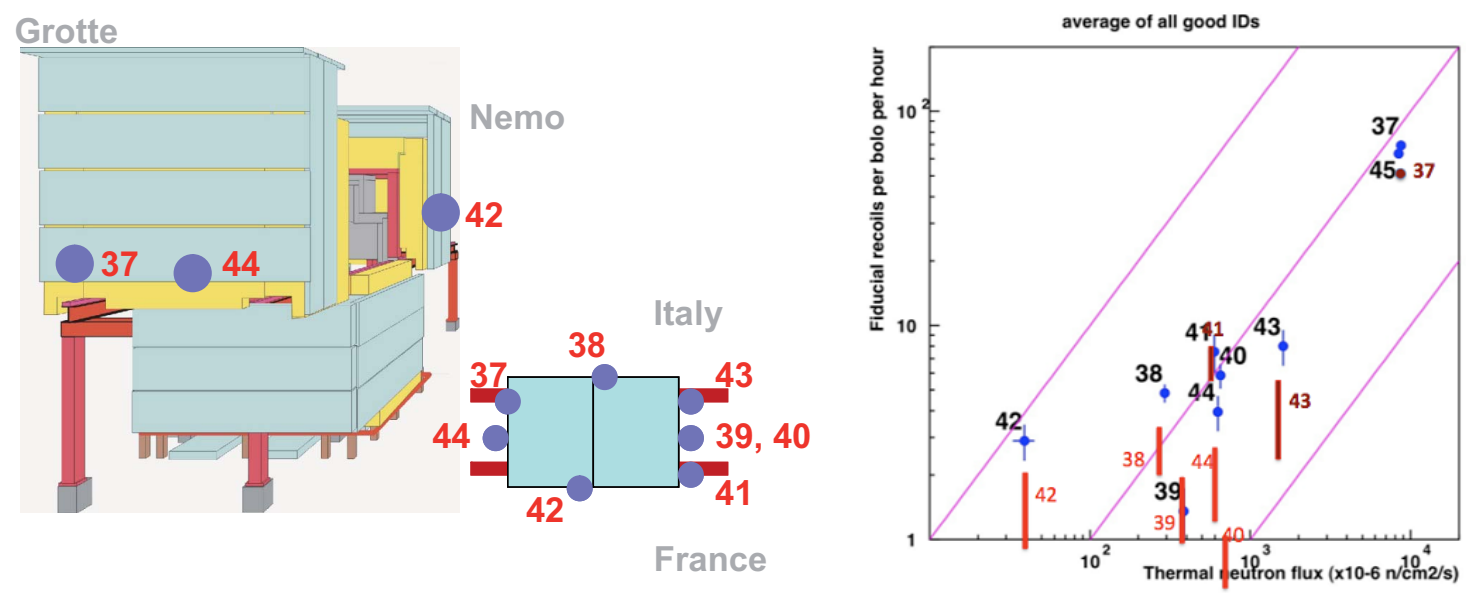

Figure 2: Left: different positions of the strong AmBe source at the EDELWEISS-2 set-up. Right: correlation of thermal neutron flux between ${ }^{3} \mathrm{He}$ detector placed near the cryostat and Ge-bolometers of EDELWEISS; in red are simulated data (GEANT4). 
One ${ }^{3} \mathrm{He}$-gas detector was installed inside the shields (Fig. 1) during the EDELWEISS physics run for dark matter search in 2009-2010. Preliminary analysis of data acquired within 274 live days yields a thermal neutron flux of $\Phi_{\text {inside }}=(7.3 \pm 1.8) 10^{-9} \mathrm{~cm}^{-2} \mathrm{~s}^{-1}$, thus showing a reduction by at least three orders of magnitude comparing to the results obtained outside the shields. The same detector was also used during a special measurement with a strong AmBe source ( $2 \cdot 10^{5}$ neutrons/s) in March 2010. The data collected with Ge-bolometers and the ${ }^{3} \mathrm{He}$ proportional counter allowed to improve our understanding and verify the efficiency of the EDELWEISS-2 polyethylene shield through comparison with dedicated GEANT4 simulations (Fig. 2). These simulations generally confirmed that $50 \mathrm{~cm}$ of polyethylene attenuate a fast neutron flux by factor of $10^{5 \div 6}$.

\section{Measurement of muon-induced neutrons}

LSM laboratory is shielded by 4850 m.w.e. of rock which reduces a muon flux down to about $5 \mathrm{~m}^{-2} \mathrm{day}^{-1}$. Nevertheless, the remaining muons can still cause valuable background for dark matter search by inducing neutron production in either high-Z shielding materials (e.g. lead) or in a nearby rock. A muon veto around the experiment helps to reduce this background significantly. However, neutrons produced in the rock by muons passing outside of the set-up and hence not tagged by the veto, may still reach the dark matter detectors. In addition, fraction of muons can be missed due to a non-ideal efficiency of the veto itself. In order to study the effect of this muon-induced background, GEANT4 simulations with the full event topology were developed [6]. Results of the simulations were compared with the analysis of muon veto - bolometer coincidence events and rather good agreement was found [7]. Unfortunately, such data suffer from rather low statistics, e.g. only a few muoninduced neutrons could be observed within $\sim 100$ live days. This is why in order to further tune the background model, a dedicated neutron counter based on $1 \mathrm{~m}^{3}$ of Gd-loaded liquid scintillator was developed and installed in LSM in fall 2008 [8]. A neutron capture on $\operatorname{Gd}\left(\sigma_{\gamma}=2.54 \cdot 10^{5}\right.$ barn for ${ }^{157} \mathrm{Gd}$ ) results in several $\gamma$ 's with a summed energy of $8 \mathrm{MeV}$. The volume of the scintillator is viewed by 16 PMTs of 8 -inch diameter ( 8 on each side) optimized for neutron capture events and by 6 PMTs of 2-inch diameter for a muon detection. A 10 -cm thick lead layer is put underneath the scintillator to enhance the neutron production. A plastic muon veto module of the same type as the main EDELWEISS veto is placed on top of the neutron counter. There are two signatures of muon-induced neutrons, both based on a detection of a multi-hit event within the acquisition window $\mathrm{T}_{D A Q} \sim 60 \mu \mathrm{s}$ : a) first, a coincidence between the muon veto module on top of the neutron counter and the neutron counter itself is identified and then secondary hits in the neutron counter data are searched for; $b$ ) events with several hits within the $\mathrm{T}_{D A Q}$ are looked for in the neutron counter data only. In the former case the original muon is tagged while in the latter case there is no this requirement and thus events induced by high energy neutrons produced outside of the system can be detected as well. The time window of $60 \mu$ s is defined such since the capture time constant of the chosen scintillator $(0.2 \%$ of Gd by weight) is about $17 \mu \mathrm{s}$. Before going for a long physics run, the full verification of the detector was performed with a weak AmBe neutron source (20 neutrons/s). This source has an advantage that $60 \%$ of neutrons are accompanied by $\gamma$ 's of $4.4 \mathrm{MeV}$ thus resembling muon - neutron signature. The measurement showed that $\gamma$-neutron correlated events can be well identified by the detection of number of secondary hits and comparing it with an expectation of accidental coincidences background, 
by looking into the time intervals between primary hit and any secondary one and that shapes of measured energy spectra are well understood and in agreement with the GEANT4 simulations [8].
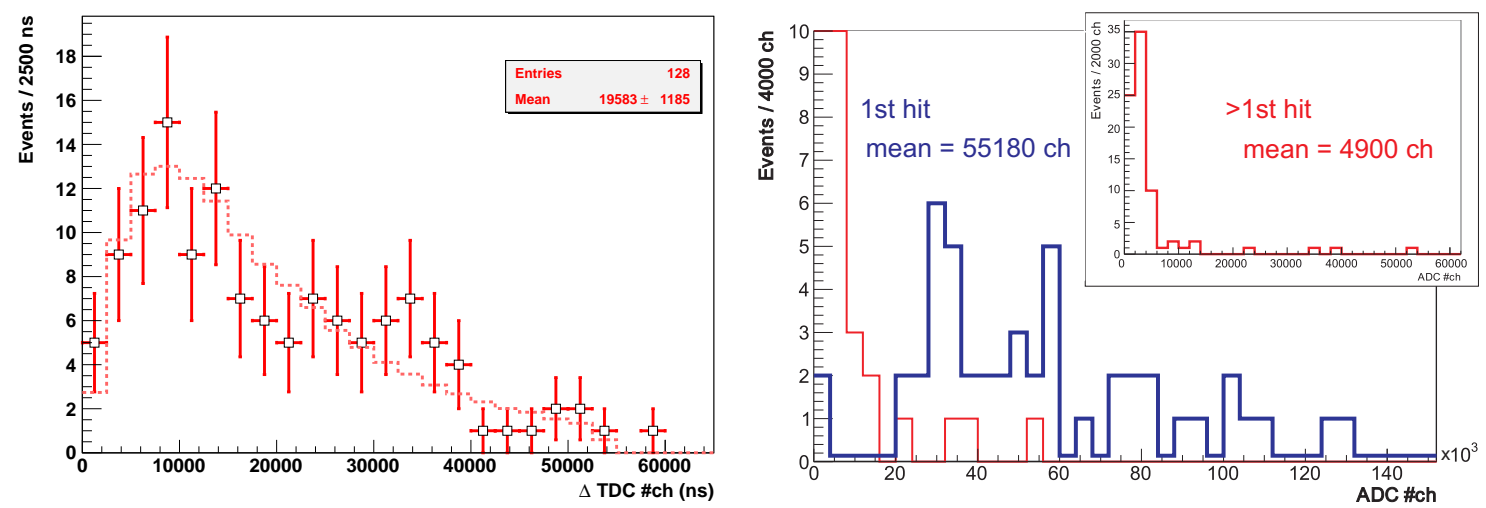

Figure 3: Left: time intervals between first and any following TDC hit, the events are in coincidence with the muon veto module on top of the neutron counter (357 live days); dash line corresponds to AmBe calibration measurement scaled down according to the number of entries. Right: energy deposited in the neutron counter when at least two hits within the same event are detected: energy spectrum of primary hits is shown in blue and the spectrum of any secondary hit is in red (right top conner).

In a long term physics run we identify with the first approach (a) 1927 muons which produced 128 secondaries in the neutron counter within 357 live days while 1.25 accidental coincidence is expected. The spectrum of time intervals between the first hit and any secondary one has the mean time of $19.6 \pm 1.2 \mu \mathrm{s}$ (Fig. 3(left)) and in agreement with the mean time of AmBe calibration, $\langle t\rangle_{A m B e}=18.5 \mu \mathrm{s}$. Furthermore, the Kolmogorov-Smirnov (KS) test is performed on unbinned data of this physics run and AmBe calibration and yields prob. $(K S)=0.42$ showing consistency of both time spectra (Fig. 3(left)). The energy distribution of primary hits is at much higher position than the one for secondary hits. This corresponds rather well to expected losses of $\mathrm{E} \sim 100 \mathrm{MeV}$ for original muons crossing the neutron counter while secondary neutrons are captured on $\mathrm{Gd}$ and thus $\mathrm{E} \sim 8 \mathrm{MeV}$ is deposited (Fig. 3(right)). With the second approach (b), i.e. without tagging the original muon, 471 secondaries produced in the neutron counter within 337 live days were identified while 34.7 accidentals are expected. The spectrum of time intervals between primary and any secondary hits shows again similar mean time of $20.5 \pm 0.7 \mu$ s (Fig. 4(left)) while KS-test yields prob. $(K S)=10^{-3}$ when unbinned data of this physics event selection and AmBe calibration are compared. This can happen if, for instance, there is a contribution from events with primary and secondary hits both produced by captured neutrons only as in this case the time distribution will differ from the one measured with the AmBe source. This is partially supported by the fact that the energy spectrum of primary hits being still at higher position than for secondaries has lower mean value than in the case of the first approach (Fig. 4(right)). This issue as well as an effect of possible backgrounds (e.g ${ }^{214} \mathrm{Bi}^{214} \mathrm{Po}$, ambient neutrons) are subject of further investigations via both more detailed analysis and simulations.

\section{Conclusion and outlook}

Ambient and muon-induced neutrons constitute a prominent background component for direct dark matter searches. The ongoing studies carried out by the EDELWEISS collaboration in this respect 

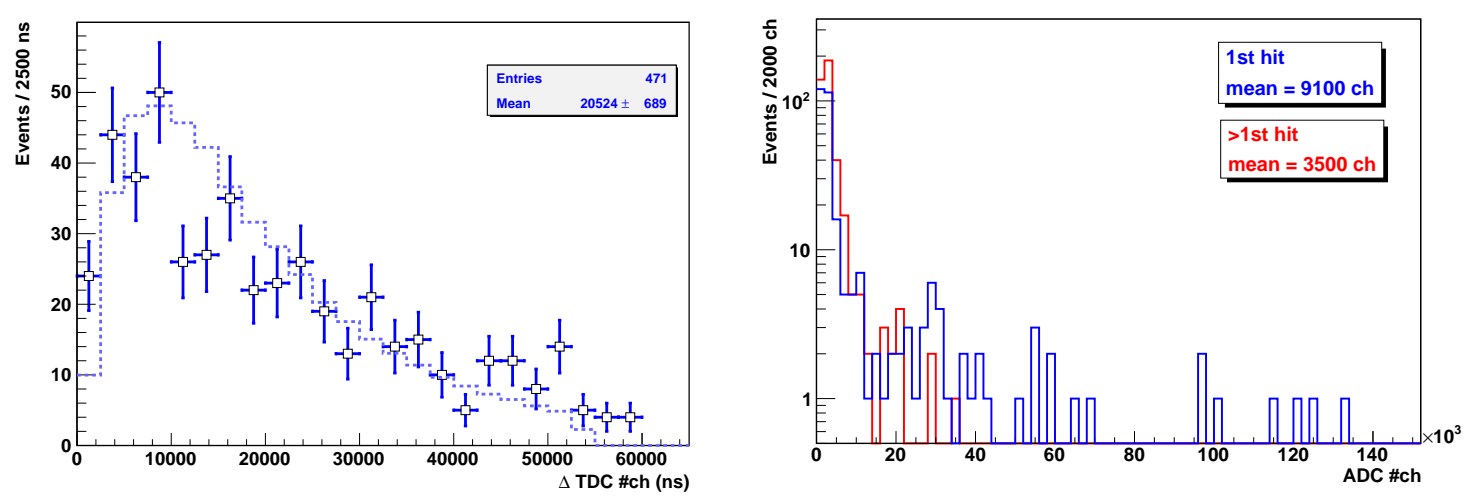

Figure 4: Left: time intervals between first and any following TDC hit, only neutron counter data and at least one secondary hit within the event is required (337 live days); dash line corresponds to AmBe calibration measurement scaled down according to the number of entries. Right: energy deposited in the neutron counter plotted for the events selected in the same way as for the time intervals: energy spectrum of primary hits is shown in blue and the spectrum of any secondary hit is in red (note the logarithmic scale).

were presented. This includes measurements with ${ }^{3} \mathrm{He}$ proportional counters at different places in the LSM underground laboratory, in particular in a proximity to the EDELWEISS set-up as well as inside the EDELWEISS shields and investigations of the muon-induced neutrons by means of GEANT4based simulations and dedicated measurement with Gd-loaded liquid scintillator detector. This wide program should lead to a development of the complete neutron background model in LSM which can then be used for a next generation dark matter experiments, e.g. EURECA.

This work is in part supported by the German Research Foundation (DFG) through the Transregional Collaborative Research Center SFB-TR27 as well as by the EU contract RII3-CT-2004-506222 and the Russian Foundation for Basic Research (grant No. 07-02-00355-a).

\section{References}

[1] E. Armengaud for the EDELWEISS Collaboration, PoS(IDM2010) 012 (these proceedings) and arXiv:1011.2319v1 [astro-ph.CO]; V.Yu. Kozlov for the EDELWEISS Collaboration, arXiv:1010.5947v1 [astro-ph.IM] (PASCOS2010, Valencia, Spain, 2010).

[2] A. Broniatowski et al., Phys. Lett. B 681, (2009) 305 and references there in, arXiv:0905.0753v1 [astro-ph.IM].

[3] H. Kraus et al., Nucl. Phys. B (Proc. Suppl.) 173, (2007) 168; PoS(IDM2010) 109 (these proceedings).

[4] V. Chazal et al., Astropart. Phys. 9 (1998) 163.

[5] S. Rozov et al., arXiv:1001.4383v1 [astro-ph.IM].

[6] M. Horn, Simulations of the muon-induced neutron background of the EDELWEISS-II experiment for Dark Matter search, Ph.D. thesis, Universität Karlsruhe (TH), 2007 and FZKA scientific report 7391 (http://bibliothek.fzk.de/zb/berichte/FZKA7391.pdf)

[7] A. Chantelauze, Investigation of the muon-induced background of the EDELWEISS-II experiment, Ph.D. thesis, Université Blaise Pascal, Clermont-Ferrand, 2009.

[8] V.Yu Kozlov et al., Astropart. Phys. 34 (2010) 97; arXiv:1006.3098v1 [astro-ph.IM]. 